\title{
Resistencia a metronidazol y claritromicina en aislamientos de Helicobacter pylori de pacientes dispépticos en Colombia
}

\author{
Adalucy Álvarez ${ }^{1 a}$, José Ignacio Moncayo ${ }^{1 b}$, \\ Jorge Javier Santacruz ${ }^{1 b}$, Luisa Fernanda Corredor ${ }^{1 c}$, \\ Elizabeth Reinosald, José W illiam Martínez ${ }^{2}$, \\ Leonardo Beltrán 3 e. \\ Antimicrobial susceptibility of \\ Helicobacter pylori strains isolated \\ in Colombia
}

Background: Helicobacter pylori antimicrobial resistance rates differ among countries and even between different areas of a country. In Colombia, the most commonly used antimicrobials for the treatment of $\mathrm{H}$ pylori infection are amoxicillin, clarithromycin and metronidazole. Aim: To determine antimicrobial susceptibility of $\mathrm{H}$ pylori strains isolated in Colombia. Materials and methods: Eighty eight strains of $\mathrm{H}$ pylori were isolated and identified by microbiological methods and confirmed with polymerase chain reaction (PCR). The detection of antimicrobial resistance to amoxicillin, clarithromycin, metronidazole and tetraclycline, was conducted by the Etest method. Mutations in the 23S rDNA, involved in resistance to clarithromycin, were detected using PCR and restriction fragment lenght polymorphism. Results: Eighty eight and 2.2\% of the strains were resistant to metronidazole and clarithromycin, respectively. No isolate was simultaneously resistant to amoxicillin or tetracycline. The two clarithromycin resistant strains were homozygous for the A2143G mutation. No mutations were found in the remaining 86 susceptible strains. Conclusions: The high rate of metronidazole resistance in our population precludes the use of this drug for the empirical treatment of $H$ pylori infection (Rev Méd Chile 2009; 137: 1309-14). (Key w ords: Antibacterial agents; Helicobacter pylori; Metronidazole)

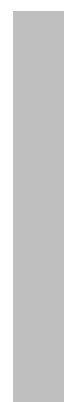

\footnotetext{
Recibido el 29 de enero, 2009. Aceptado el 8 de septiembre, 2009.

Trabajo financiado por la Vicerrectoría de Investigaciones, Innovación y Extensión de la Universidad Tecnológica de Pereira, Colombia.

${ }^{1}$ Laboratorio de Microbiología y Parasitología, Centro de Biología Molecular y Biotecnología, Departamento de Ciencias Básicas, Facultad de Ciencias de la Salud, Universidad Tecnológica de Pereira, Pereira, Colombia. ${ }^{2}$ Departamento de Medicina Comunitaria y ${ }^{3}$ Laboratorio de Genética Médica, Facultad de Ciencias de la Salud, Universidad Tecnológica de Pereira, Pereira, Colombia. a Bacterióloga, MSc en Biología Molecular y Biotecnología

bLicenciado en Biología, MSc en Microbiología

${ }^{\mathrm{C}}$ Licenciada en Biología y Química, MSc en Biología Molecular y Biotecnología

${ }^{\mathrm{d}}$ Estudiante X semestre de Medicina

eQuímico Industrial
}

Correspondencia a: Adalucy Álvarez Aldana. Universidad Tecnológica de Pereira, Facultad de Medicina, Laboratorio de Microbiología y Parasitología. Vereda La Julita. Pereira, Colombia. E mail: adalucy@yahoo.es /adalucyalvarez@utp.edu.co 
$\mathrm{L}$ a infección por Helicobacter pylori es una de las más comunes en el mundo, causa gastritis y enfermedad úlcero-péptica y se asocia con carcinoma gástrico y linfoma gástrico tipo MALT ${ }^{1}$. La prevalencia de la infección en pacientes dispépticos en Colombia es mayor a $80 \%{ }^{2}$.

Diversos estudios han demostrado que la resistencia antimicrobiana perjudica significativamente la eficacia de la terapia anti $\mathrm{H}$ pylori ${ }^{3}$. La utilización de regímenes terapéuticos basados en pruebas de sensibilidad antimicrobiana no es lo habitual $^{4}$. Esto puede atribuirse a la dificultad y al costo del aislamiento del microorganismo y de las pruebas de sensibilidad $^{5}$, por lo que la mayoría de las guías publicadas para el manejo de la infección por $\mathrm{H}$ pylori recomiendan tratamiento empírico ${ }^{6}$.

A nivel mundial, la terapia más utilizada es triple y consiste en un inhibidor de la bomba de protones (IBP), más dos antibióticos ${ }^{7}$. En Colombia, los antibióticos más prescritos para el tratamiento de $\mathrm{H}$ pylori son amoxicilina (73\%), claritromicina $(57 \%)$ y metronidazol $(37 \%)^{8}$.

El aumento en la prevalencia de la resistencia complica seriamente la erradicación de la infección y esta prevalencia varía geográficamente, aun dentro de un mismo país ${ }^{3}$. En Colombia, la resistencia reportada para el metronidazol es de $86 \%$, mientras que para otros antimicrobianos no ha sido publicada 8 .

Los métodos fenotípicos reportados para la detección de la resistencia en $\mathrm{H}$ pylori son: dilución en agar, dilución en caldo, breakpoint en agar, difusión con disco y Etest ${ }^{9}$. El método de dilución en agar es considerado el método de referencia por el Clinical and Laboratory Standards Institute (CLSI). El método de Etest es una de las mejores alternativas disponibles al de dilución en agar y ha demostrado correlaciones que van desde $86 \%$ a $99,5 \%$ con el método de referencia ${ }^{10,11}$. Tiene la ventaja de ser un método comercial cuantitativo, fácil de ejecutar e interpretar.

Numerosos métodos genotípicos se han desarrollado para detectar resistencia a claritromicina, debido al impacto de esta resistencia y al bajo número de mutaciones involucradas ${ }^{3}$. La resistencia a la claritromicina se fundamenta en mutaciones puntuales situadas en el ARNr 23S. La mayoría de los aislamientos resistentes contienen la mutación A2143G (69,8\%) o A2142G (11,7\%). Mientras que la mutación A2142C sólo representa de 0\% a $7 \%$ y existen otras menos frecuentes como la T2717C y la T2182C $3,9,12$.
El presente estudio se realizó para detectar: (a) La prevalencia de la resistencia a los antimicrobianos metronidazol, claritromicina, amoxicilina $y$ tetraciclina en aislamientos de $\mathrm{H}$ pylori, obtenidos de pacientes dispépticos de Pereira y Armenia, (b) la distribución de la concentración inhibitoria mínima (CIM) en las cepas de $\mathrm{H}$ pylori con estos antimicrobianos, y (c) las dos mutaciones más frecuentes involucradas en la resistencia a claritromicina.

\section{MATERIAL Y MÉTODO}

Población de estudio. Un total de 88 cepas de $\mathrm{H}$ pylori obtenidas de pacientes con síntomas dispépticos que asistieron a consulta a unidades de endoscopia en las ciudades de Pereira y Armenia, y aceptaron participar por escrito firmando el consentimiento informado, durante los años 2000, 2003 y 2006. Esta investigación obtuvo aprobación del Comité de Bioética de la Facultad de Ciencias de la Salud, de la Universidad Tecnológica de Pereira.

Cultivos de $\mathrm{H}$ pylori. Las cepas de $\mathrm{H}$ pylori fueron identificadas usando métodos convencionales (morfología de la colonia, coloración de Gram y pruebas bioquímicas como oxidasa, catalasa y ureasa) y uno no convencional (PCR $)^{2}$. Los aislamientos fueron almacenados en caldo tripticasa soya con glicerol al $20 \%$ a $-80^{\circ} \mathrm{C}$ hasta el momento de ser utilizados y en amortiguador TE a $-20^{\circ} \mathrm{C}$ para la extracción del ADN.

Detección de resistencia en las cepas de $\mathrm{H}$ pylori.

1. Método fenotípico por Etest para la detección de la resistencia a metronidazol, claritromicina, amoxicilina y tetraciclina:

Cada una de las cepas de $\mathrm{H}$ pylori se cultivaron durante 2 días en TSA-sangre. Los cultivos fueron suspendidos en caldo Brucella hasta lograr una turbidez de McFarland 3. La suspensión fue inoculada en cinco placas de agar Mueller Hinton con sangre, una placa por cada tirilla del antimicrobiano a tamizar y la quinta placa fue el control de viabilidad del aislamiento. Las placas se incubaron durante 3 días a $37^{\circ} \mathrm{C}$ en una atmósfera microaerofílica $\left(5 \%\right.$ de $\mathrm{O}_{2}$, el $10 \%$ de $\mathrm{CO}_{2}$ y $85 \% \mathrm{~N}_{2}$ ) (Water Jacketed Incubator, TS Autoflow $\mathrm{CO}_{2} / \mathrm{O}_{2}$, NUAIRE). La actividad antimicrobiana se observó como un halo de inhibición 
de crecimiento que permite visualizar directamente la CIM, por la escala numérica presente en la tirilla, en el punto donde la elipse cruza con la escala (AB Biodisk, EAS 013). En el presente estudio, el punto de corte para establecer la resistencia a metronidazol, claritromicina, amoxicilina y tetraciclina, se definió como $>4,0$, $\geq 1,>1$ y $>2 \mathrm{mg} / \mathrm{L}$, respectivamente (AB Biodisk, EAS 013). La cepa de H pylori NCTC 11637 fue utilizada como cepa control.

2. Método genotípico por PCR-RFLP para la detección de resistencia a la claritromicina:

A partir del ADN genómico extraído con el kit Wizard $^{\circledR}$ Genomic DNA purification (Promega, Cat A1120), se realizaron PCRs de las 88 cepas con la pareja de iniciadores (HPYS 5'- AGG TTA AGA GGA TGC GTC AGT C -3' y HPYA 5'- CGC ATG ATA TTC CCA TTA GCA GT -3') (número de acceso Gen Bank 881379) ${ }^{13}$, para amplificar un fragmento de $267 \mathrm{pb}$ del ARN ribosomal 23S. La reacción de amplificación se efectuó en un volumen final de $50 \mu \mathrm{l}$, conteniendo buffer 1X, $\mathrm{MgCl}_{2} 1,5 \mathrm{mM}$, dNTPs $200 \mu \mathrm{M}$, iniciadores 10 pmoles de cada uno, Taq polimerasa (Promega, buffer A) $1.25 \mathrm{U}$ y ADN bacteriano $2 \mu \mathrm{l}$. En un termociclador Perkin Elmer GeneAmp 9700, se utilizaron 35 ciclos de $95^{\circ} \mathrm{C}$ por $45 \mathrm{~s}, 60^{\circ} \mathrm{C}$ por 30 $\mathrm{s}$ y $72^{\circ} \mathrm{C}$ por un minuto. Como controles positivos se utilizaron dos cepas de nuestro laboratorio con mutaciones A2143G y A2142G, y como control negativo agua destilada estéril. Los $50 \mu \mathrm{l}$ del producto de PCR, tanto de las muestras como de los controles, fueron corridos en agarosa de bajo punto de fusión (Fisher, BP1360-100) al 3\% teñida con bromuro de etidio $(0,01 \mathrm{mg})$, con buffer TAE $1 \mathrm{X}$ a 100 voltios durante $50 \mathrm{~min}$. Las bandas de $267 \mathrm{pb}$ fueron cortadas y purificadas con el kit comercial de Promega (Wizard ${ }^{\circledR}$ PCR Preps Cat A7170) o de Amershan Biosciences (GFX PCR DNA and Gel Band Purificaction), siguiendo las instrucciones del fabricante.

La reacción de RFLP fue llevada a cabo con las enzimas de restricción BbsI (7.5U) y BsaI (15U) (New England Biolabs), utilizando $5 \mu \mathrm{L}$ del producto de PCR purificado, en un volumen final de $15 \mu \mathrm{L}$ a la temperatura sugerida por el fabricante, durante toda la noche. Los fragmentos de restricción, se comprobaron corriendo en gel de acrilamida al 12\%, teñido con bromuro de etidio, con buffer TBE $1 \mathrm{X}$ a 230 voltios.
Todos los aislamientos con mutación por PCRRFLP se secuenciaron con el kit BigDye Terminador V3.1. La reacción de secuencia se preparó utilizando por cada muestra $4 \mu \mathrm{L}$ de Terminator Ready Reaction Mix, 12 pmoles del iniciador HPY$S$ o HPY-A y $4 \mu \mathrm{L}$ del producto de PCR HPY-S y HPY-A purificado por los kits comerciales descritos anteriormente. La amplificación se realizó en un termociclador Perkin Elmer GeneAmp 9700 con las siguientes condiciones: 35 ciclos a $96^{\circ} \mathrm{C}$ por $10 \mathrm{~s}, 50^{\circ} \mathrm{C}$ por $5 \mathrm{~s} \mathrm{y} 60^{\circ} \mathrm{C}$ por $4 \mathrm{~min}$. Este producto de amplificación fue purificado por el método de precipitación con Etanol/EDTA/Acetato, siguiendo las instrucciones del fabricante (kit BigDye Terminator V3.1). El botón se resuspendió en $10 \mu \mathrm{L}$ de formamida y fue corrido en el ABI Prism 3100 Avant (Applied Biosystems), utilizando un capilar de $36 \mathrm{~cm}$ y el polímero POP4 (Performance Optimized Polymer, Applied Biosystems). Los datos fueron analizados en el software Sequencing Analysis 5.1. Como control se sometieron a secuencia dos aislamientos que no presentaron mutaciones por el método PCR-RFLP.

\section{Resultados}

Detección de resistencia en las cepas de H pylori:

1. Método fenotípico por Etest para la detección de la resistencia a metronidazol, claritromicina, amoxicilina y tetraciclina:

De las 88 cepas ninguna fue resistente a tetraciclina y amoxicilina, 2,2\% (2/88) fueron resistentes a claritromicina y $88 \%$ (68/88) fueron resistentes a metronidazol. Las dos cepas resistentes a claritromicina fueron simultáneamente resistentes a metronidazol. En la Tabla 1, se observan los datos de la distribución de las CIMs para metronidazol, claritromicina, amoxicilina y tetraciclina. Las dos cepas resistentes a claritromicina presentaron CIMs de 16 y 32 mg/L. La distribución de las CIMs en las cepas resistentes a metronidazol fue variable, pero la mayoría presentaban CIMs $\geq 256 \mathrm{mg} / \mathrm{L}$.

2. Método genotípico por PCR-RFLP para la detección de resistencia a claritromicina:

Todos los ADN extraídos de las 88 cepas de $\mathrm{H}$ pylori que se sometieron a PCR utilizando los iniciadores (HPY-S y HPY-A) amplificaron un fragmento de $267 \mathrm{pb}$. De estas 88 cepas sometidas a restricción, en dos $(2,2 \%)$ se produjeron 
Tabla 1. D istribución de los CIM s en las 88 cepas de H pylori

\begin{tabular}{|lcccc|}
\hline Etest & Metronidazol & Claritromicina & Amoxicilina & Tetraciclina \\
CIM $\mathbf{~ m g / L ~}$ & $1(1,13)$ & $58(65,9)$ & $63(71,6)$ & $7(7,95)$ \\
& $1(1,13)$ & $24(27,2)$ & $22(25)$ & $68(77,2)$ \\
$0,023-0,0,094$ & $2(2,27)$ & $4(4,54)$ & $3(3,4)$ & $13(14,7)$ \\
$0,125-0,19$ & $4(4,54)$ & - & - & - \\
$0,5-0,75$ & $2(2,27)$ & - & - & - \\
$1-2$ & $1(1,13)$ & - & - & - \\
$6-8$ & $5(5,68)$ & $2(2,27)$ & - & - \\
$16-32$ & $5(5,68)$ & - & - & - \\
$48-128$ & $67(76,1)$ & - & - & - \\
$>256$ & & & & \\
\hline
\end{tabular}

cortes del producto amplificado, con la enzima de restricción BsaI, originando fragmentos de 208 y 59 pb, indicando la mutación A2143G. Mientras que con la enzima BbsI no se presentaron cortes, por lo que la mutación A2142G no fue detectada en ninguna de las cepas (Figura 1). Los resultados obtenidos por PCR-RFLP fueron confirmados con la secuenciación del producto de PCR de $267 \mathrm{pb}$, en las dos cepas que presentaron la mutación A2143G y en las dos cepas controles que no presentaron mutación.

\section{DISCUSIÓN}

Los reportes sobre tasas de resistencia de $\mathrm{H}$ pylori en Colombia son escasos, excepto por un estudio publicado en $1998^{8}$, que mostró una tasa de resistencia a metronidazol de 86\%. Nuestros resultados (88\%) confirman el anterior informe, y es similar a lo reportado para países en vías de desarrollo ${ }^{3,14,15}$. En dichas poblaciones, la resistencia a metronidazol se asocia con el uso frecuente de este fármaco para el tratamiento de parasitosis e infecciones ginecológicas $3,15,16$, y esa situación puede ser la que contribuye a la alta tasa de resistencia en nuestro país. Además, en Colombia, el metronidazol es parte del régimen terapéutico de $\mathrm{H}$ pylori ${ }^{8}$, sugiriendo que su uso también contribuiría a la resistencia. De los aislamientos resistentes a metronidazol, $76 \%$ presentaron un $\mathrm{CIM} \geq 256 \mathrm{mg} / \mathrm{L}$, este alto nivel de CIM ya ha sido reportado, pero rara vez en la mayoría de las cepas resistentes ${ }^{17}$.

La tasa de resistencia a la claritromicina detectada en este estudio $(2,2 \%)$, se asemeja a los datos de Paraguay (2\%) y la VIII región de Chile (2,2\%), pero difieren de los datos de Ecuador (9,5\%), Brasil (9,8\%), México (25\%) y de la región metropolitana de Chile $(20 \%)^{14,15,18-21}$. Lo que confirma que existe una alta variabilidad geográfica con respecto a las tasas de resistencia, aun en países latinoamericanos. La baja tasa de resistencia encontrada para la claritromicina podría ser explicada por un bajo consumo, debido a que este antimicrobiano no se encuentra dentro de los medicamentos del Plan Obligatorio de Salud. Aunque se ha descrito que luego de las primeras cepas resistentes a macrólidos, esta resistencia se disemina rápidamente en la población expuesta ${ }^{22,23}$.

La tasa de resistencia dual a claritromicina y metronidazol en este estudio fue baja $(2,2 \%)$, similar

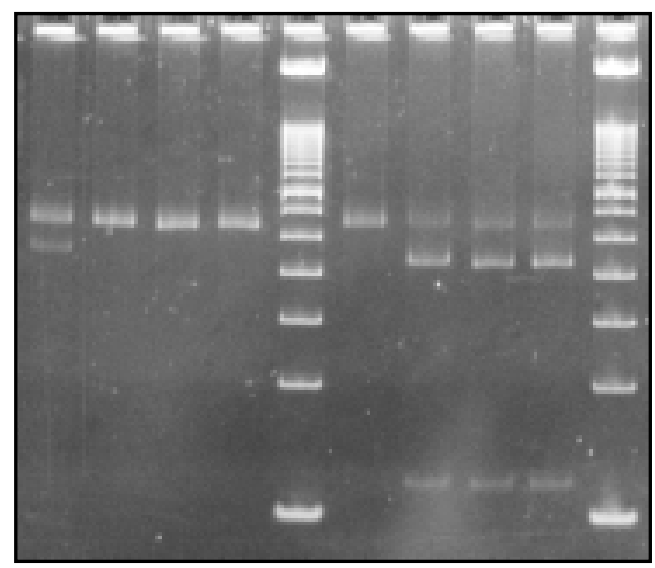

Figura 1. PCR-RFLP en gel de poliacrilamida al 12\%. Líneas 5 y 10 escalera de 50 pb. Líneas 1-4 fragmentos digeridos con BbsI. Líneas 6-9 fragmentos digeridos con BsaI. Líneas 1 y 6 cepa control con la mutación A2142G. Líneas 2 y 7 cepa control con la mutación A2143G. Líneas 3 y 8; 4 y 9 las dos cepas de $\mathrm{H}$ pylori que presentaron la mutación A2143G. 
a la descrita en Europa (0,8\%-9,1\%), en Asia (2\%-3\%) y en algunos países de Latinoamérica como Ecuador $(7,1 \%)$, Paraguay $(2,1 \%)$; pero mucho menor a la descrita en México (18\%) y Chile (13,2\%) 3,15,18,21. Cuando existe resistencia a ambos antimicrobianos, los ensayos clínicos han descrito que la infección no se erradica, reforzando la preocupación del uso de esta combinación como primera línea de tratamiento, ya que alrededor de $50 \%$ de las cepas pueden adquirir la doble resistencia en pacientes en donde falla la terapia de erradicación ${ }^{3}$.

No se encontraron aislamientos con resistencia a amoxicilina y tetraciclina, similar a lo descrito por otros autores en otras partes del mundo, donde las tasas de resistencia son $\leq 1 \%$ para la amoxicilina $\mathrm{y} \leq 5,3 \%$ para la tetraciclina ${ }^{20,24,25}$. Sin embargo, existen casos excepcionales en donde se han reportado tasas de resistencia a amoxicilina de $72 \%$ y tetraciclina de $59 \%$.

Los resultados de resistencia a claritromicina por PCR-RFLP y Etest confirman la excelente correlación que existe entre la determinación del CIM usando Etest y la detección de mutaciones en el ARNr 23S por PCRRFLP. Adicionalmente, el método de PCR-RFLP tiene la ventaja de poderse aplicar directamente en muestras de biopsia gástrica y materia fecal, sin necesidad de aislar previamente el microorganismo ${ }^{13,27}$.

En el ADN genómico de $\mathrm{H}$ pylori existen dos copias del ARNr 23S, la mutación en una copia es suficiente para conferir resistencia a claritromicina ${ }^{28,29}$. La secuenciación mostró que las mutaciones encontradas por PCR-RFLP estuvieron presentes en ambas copias de las dos cepas resistentes a claritromicina.

Se ha comunicado que el nivel del CIM varía de acuerdo con el tipo de mutación, la mutación A2142G se asocia con CIMs $\geq 64 \mathrm{mg} / \mathrm{L}$ y la

\section{REFERENCIAS}

1. Kusters J, Vliet A, Kuipers E. Pathogenesis of Helicobacter pylori infection. Clin Microbiol Rev 2006; 19: 449-90.

2. Moncayo Ji, Santacruz JJ, Álvarez al, Franco B, López MA, Ángel A et AL. Comparación de métodos diagnósticos en la infección por Helicobacter pylori en Quindío, Colombia. Colomb Méd 2006; 37: 203-12.

3. Megraud F. H pylori antibiotic resistance: prevalence, importance, and advances in testing. Gut 2004; 53: $1374-84$

4. Datta S, Chattopadhyay S, Patra R, De R, Ramamurthy T, Hembram J et al. Most Helicobacter pylori strains of Kolkata in India are resistant to metronidazole but susceptible to other drugs commonly used for eradication and ulcer mutación A2143G con CIMs $\leq 64 \mathrm{mg} / \mathrm{L}^{30,31}$. Nuestros resultados para la mutación A2143G (CIMs de 16 y $32 \mathrm{mg} / \mathrm{L}$ ), son similares a lo descrito. En este estudio, fue más frecuente la mutación A2143G al igual como se reporta a nivel mundial $31-33$.

En conclusión, este es el primer estudio de resistencia antimicrobiana que se realiza en cepas de $\mathrm{H}$ pylori obtenidas de pacientes dispépticos de las ciudades de Pereira y Armenia. Igualmente, es la primera vez que se hace un reporte de cepas resistentes en estas ciudades. La alta tasa de resistencia a metronidazol encontrada en este estudio, precluye el uso de este antimicrobiano en el tratamiento empírico de la infección por H pylori en esta población. Los otros tres antimicrobianos tamizados no presentan inconvenientes para su utilización debido a su baja o nula resistencia.

Es necesario que en todo el país se realice una vigilancia epidemiológica de la evolución de la resistencia en esta especie, ya que es necesario conocer qué ocurre en este aspecto en cada lugar geográfico del país, para orientar los esquemas de tratamiento. Nuestro grupo de investigación ya se encuentra estudiando aislamientos de la ciudad de Manizales, para completar un perfil de la resistencia en la región del Eje Cafetero. Además, los datos de un país en desarrollo como Colombia, contribuyen al conocimiento de las estadísticas epidemiológicas mundiales de la resistencia del $\mathrm{H}$ pylori.

\section{Agradecimientos}

Los autores expresan su agradecimiento a la Universidad Tecnológica de Pereira, al Centro de Biología Molecular y Biotecnología y al Laboratorio de Genética Médica. Igualmente, al señor Arcángel de Jesús Mesa.

therapy. Aliment Pharmacol Ther 2005; 22: 51-7.

5. Toracchio S, Marzio L. Primary and secondary antibiotic resistance of Helicobacter pylori strains isolated in central Italy during the years 1998-2002. Dig Liver Dis 2003; 35: 541-5.

6. CheY W, Wong B. American College of Gastroenterology Guideline on the Management of Helicobacter pylori Infection. Am J Gastroenterology 2007; 102: 1808-25.

7. Abbles AZ, Simon I, Melton ER. Update on Helicobacter pylori Treatment. Am Fam Physician 2007; 75: 351-8.

8. Gómez M, Otero W, Gutiérrez O. Tratamiento de la infección por Helicobacter pylori en un grupo de médicos generales y especialistas en Colombia. Rev Col Gastroenterol 2007; 22: 7-16.

9. Megraud F, Lehours P. Helicobacter pylori detection 
and antimicrobial susceptibility testing. Clin Microbiol Rev 2007; 20: 280-322.

10. Glupczynski Y, Broutet N, Cantagrel A, Andersen LP, Alarcón T, López Brea M et al. Comparison of the Etest and agar dilution method for antimicrobial suceptibility testing of Helicobacter pylori. Eur J Clin Microbiol Infect Dis 2002; 21: 549-52.

11. Best L, Haldane DJ, Keelan M. Multilaboratory comparison of proficiencies in susceptibility testing of Helicobacter pylori and correlation between agar dilution and Etest methods. Antimicrob Agents Chemother 2003; 47: 3138-44.

12. Raymond JC, Burucoa O, Pietrini M, Bergeret A, Decoster A, Wann C ET al. Clarithromycin resistance in Helicobacter pylori strains isolated from French children: prevalence of the different mutations and coexistence of clones harboring two different mutations in the same biopsy. Helicobacter 2007; 12: 157-63.

13. Menard A, Santos A, Megraud F, Oleastro M. PCRrestriction fragment length polymorphism can also detect point mutation $\mathrm{A} 2142 \mathrm{C}$ in the $23 \mathrm{~S}$ rRNA gene, associated with Helicobacter pylori resistance to clarithromycin. Antimicrob Agents Chemother 2002; 46: 1156-8.

14. Debets-Ossenkopp YJ, Reyes G, Mulder J, Stegge BM, Peters JTAM, Savelkoul PHM et al. Characteristics of clinical Helicobacter pylori strains from Ecuador. J Antimicrob Chemother 2003; 51: 141-5.

15. Torres J, Camorlinga-Ponce M, Pérez-Pérez G, Madrazo de la Garza A, Dehesa M, González-Valencia G ET AL. Increasing multidrug resistance in Helicobacter pylori strains isolated from children and adults in México. J Clin Microbiol 2001; 39: 2677-80.

16. McMahon BJ, Hennessy TW, Bensler JM, Bruden DL, PARKINSON AJ, Morris JM ET al. The relationship among previous antimicrobial use, antimicrobial resistance, and treatment outcomes for Helicobacter pylori infections. Ann Intern Med 2003; 139: 463-9.

17. Albert J, Al-Merhaizeem K, Neil L, Dhar R, Dhar PM, AL-Ali M ET AL. High prevalence and level of resistance to metronidazole, but lack of resistance to other antimicrobials in Helicobacter pylori, isolated from a multiracial population in Kuwait. Aliment Pharmacol Ther 2006; 24: 1359-66.

18. Farina N, Kasamatsu E, Samudio M, Morán M, Sanabria R, Laspina F. Susceptibilidad a antibióticos de cepas paraguayas de Helicobacter pylori aisladas de pacientes con enfermedad gastroduodenal. Rev Méd Chile 2007; 135: 1009-14.

19. González C, García A, Daroch F, Kawaguchi F, Solar H, Rivera N ET al. Susceptibilidad in vitro de cepas de Helicobacter pylori: aislamiento de cepas resistentes a claritromicina. Rev Méd Chile 2001; 129: 643-6.

20. Mendoca S, Ecclissato C, Sartori MS, Godoy AP, Guerzoni RA, Degger M et al. Prevalence of Helicobacter pylori resistance to metronidazole, clarithromycin, amoxicillin, tetracycline, and furazolidone in Brazil. Helicobacter 2000; 5: 79-83.
21. Vallejos C, Garrido L, Cáceres D, Madrid AM, Defilippi C, Defilippi C et al. Prevalencia de la resistencia a metronidazol, claritromicina y tetraciclina en Helicobacter pylori aislado de pacientes de la Región Metropolitana. Rev Méd Chile 2007; 135: 287-93.

22. Mohammadi M, Doroud D, Massarrat S. Clarithromycin resistance in Iranian $\mathrm{H}$ pylori strains before introduction of clarithromycin. Helicobacter 2003; 8: 79-80.

23. Taneike I, Goshi SM, Tamura Y. Emergence of clarithromycin-resistant Helicobacter pylori (CRHP) with a high prevalence in children compared with their parents. Helicobacter 2002; 7: 297-305.

24. Dailidiene D, Bertoli MT, Miciuleviciene J, Mukhopadhyay AK, Dailide G, Pascasio MA et al. Emergence of tetracycline resistance in Helicobacter pylori: multiple mutational changes in $16 \mathrm{~S}$ ribosomal DNA and other genetic loci. Antimicrob Agents Chemother 2002; 46: 3940-6.

25. Dore MP, Graham DY, Sepúlveda AR. Different penicillin-binding protein profiles in amoxicillin-resistant Helicobacter pylori. Helicobacter 1999; 4: 154-61.

26. Wu H, ShI X, WANG HT, LIU JX. Resistance of Helicobacter pylori to metronidazole, tetracycline and amoxycillin. J Antimicrob Chemother 2000; 46: 121-3.

27. Rimbara E, Noguchi N, Yamaguchi T, Narui K, Kawai T, SASATSU M. Development of a highly sensitive method for detection of clarithromycin-resistant Helicobacter pylori from feces. Curr Microbiol 2005; 51: 1-5.

28. Van Doorn LJ, Glupczynski Y, Kusters JG, Mégraud F, Midolo P, Maggi-Solca N ET Al. Accurate prediction of macrolide resistance in Helicobacter pylori by a PCR line probe assay for detection of mutations in the $23 \mathrm{~S}$ rRNA gene: multicenter validation study. Antimicrob Agents Chemother 2001; 45: 1500-4.

29. Hulten K, Gibreel A, SkÖld O, Engstrand L. Macrolide resistance in Helicobacter pylori: mechanism and stability in strains from clarithromycin-treated patients. Antimicrob Agents Chemother 1997; 41: 2550-3.

30. Versalovic J, Osato MS, Spakovsky K, Dore MP, Reddy R, Stone GG et al. Point mutations in the rRNA of Helicobacter pylori associated with different levels of clarithromycin resistance. J Antimicrob Chemother 1997; 40: 283-6.

31. Alarcón T, Vega Ae, Domingo D, Martínez MJ, LópezBREA M. Clarithromycin resistance among Helicobacter pylori strains isolated from children: prevalence and study of mechanism of resistance by PCRrestriction fragment length polymorphism analysis. J Clin Microbiol 2003; 41: 486-99.

32. Domingo D, Alarcón T, Sanz JC, Sánchez I, López-Brea M. High frequency of mutations at position 2144 of the $23 \mathrm{~S}$ rRNA gene in clarithromycin-resistant Helicobacter pylori strains isolated in Spain. J Antimicrob Chemother 1998; 41: 573-4.

33. Burucoa C, Landron C, Garnier M, Fauchere JL, Khan R, RAHMAN M. T2182C mutation is not associated with clarithromycin resistance in Helicobacter pylori. Antimicrob Agents Chemother 2005; 49: 868-70. 